\title{
Audiencia en redes sociales de los debates televisados en las elecciones generales de abril de 2019
}

\section{Social media audience of televised debates in the general elections of April 2019}

Julia Fontenla-Pedreira. Universidad de Vigo. España.

julia.fontenla.pedreira@uvigo.es

$[\mathrm{CV}]$ (1)

José Rúas-Araújo. Universidad de Vigo. España.

joseruas@uvigo.es

$[\underline{\mathrm{CV}}]$ (1)

Erica Conde-Vázquez. Universidad de Vigo. España.

erikaconde@uvigo.es

[CV]

Este artículo forma parte de los trabajos desarrollados en el marco del proyecto de investigación: "DEBATv, Debates Electorales Televisados en España: Modelos, Proceso, Diagnostico y Propuesta" (CSO2017-83159$R$ ), proyecto de $I+D+I$ (Retos) financiado por el Ministerio de Ciencia, Innovación y Universidades y la Agencia Estatal de Investigación (AEI) del Gobierno de España, con el apoyo del Fondo Europeo de Desarrollo Regional (FEDER) de la Unión Europea (UE).

Cómo citar este artículo / Referencia normalizada

Fontenla-Pedreira, J., Rúas-Araújo, J. y Conde-Vázquez, E. (2020). Audiencia en redes sociales de los debates televisados en las elecciones generales de abril de 2019. Revista Latina de Comunicación Social, (76), 1-16. https://www.doi.org/10.4185/RLCS-2020-1434

\begin{abstract}
RESUMEN
Introducción: Se analiza el impacto en medios tradicionales y nuevos medios de los dos debates electorales televisados celebrados con motivo de las últimas elecciones generales de España de abril de 2019, en RTVE y Atresmedia, los días 22 y 23 de abril, respectivamente. Metodología: Se realizó una encuesta telefónica, con más de 8.000 llamadas, en las ciudades de Madrid, Barcelona, A Coruña y Vigo, de las que se obtuvieron 734 respuestas completas con un margen de error sobre el total de la muestra inferior al 4\%. Resultados: Un 52\% de los encuestados visualizó alguno de los debates, de los que casi un $28 \%$ consulta información adicional. De este conjunto, un $21 \%$ lo hizo a través de redes sociales, aunque la participación activa se situó en menos de la mitad (43,2\%). Discusión y conclusiones: Existe una brecha de edad y género en el consumo de información sobre los debates televisados a través de redes sociales. Los internautas prefieren estas redes para informarse pero no participan activamente ni otorgan credibilidad a los datos ofrecidos en ellos. Los temas que suscitan mayor interés entre los usuarios de redes coinciden con los de los medios tradicionales.
\end{abstract}

PALABRAS CLAVE: comunicación política; elecciones generales; debates electorales; agenda setting; redes sociales. 


\begin{abstract}
Introduction: The impact of the two debates for the Spanish national elections in April 2019 has been analyzed. They were broadcasted on RTVE and Atresmedia on April $22^{\text {nd }}$ and $23^{\text {rd }}$ respectively. Methods: A telephone survey was conducted in Madrid, Barcelona, A Coruña and Vigo. Out of 8000 calls, 734 complete answers about the debates broadcasted on those dates were obtained. Results: $52 \%$ of the respondents have watched one debate and from them $28 \%$ have consulted further information. From this last group, $21 \%$ have used social networks, but fewer than half have actively participated (43.2\%). Discussion and conclusions: There is an age and gender gap in the consumption of information about the televised debates through social networks as well as in their use of each of them. Internet users prefer these networks to obtain information but they do not participate and do not give any credibility to the data offered during the debates. The topics drawing more interest are the same for the users of social networks and those of traditional media.
\end{abstract}

KEYWORDS: political communication; general elections; electoral debates; agenda setting; social media.

\title{
CONTENIDOS
}

1. Introducción. 1.1. Debates electorales: de la televisión a las redes sociales. 1.2. La seducción de las segundas pantallas: gustar, compartir o algo más. 1.3. Agenda temática. 2. Metodología. 3. Resultados. 3.1. Quienes han visto los debates electorales. 3.2. Quiénes consultan información sobre los debates televisados en otros medios. 3.2.1 Debates televisados en redes sociales. 4. Discusión y conclusiones. 5. Referencias bibliográficas.

\section{Introducción}

\subsection{Debates electorales: de la televisión a las redes sociales}

Los debates electorales televisados tienen interés informativo y son efectivos para la adquisición de conocimientos por parte del público (Gallego y Bernárdez, 2017). En términos de audiencia, constituyen uno de los acontecimientos más importantes en todo proceso electoral, alcanzando históricas cuotas de pantalla (Webster, Phalen y Lichty, 2014; Berrocal, 2005; Huertas, 2002), propias de estos acontecimientos también considerados, en ocasiones, como históricos (Téllez, Muñiz y Rodríguez, 2010)

Internet y las redes sociales han provocado una metamorfosis en el consumo de información, dando paso a la denominada "sociedad red" (Castells, 2009), caracterizada por el consumo de un amplio volumen de información en un mundo hiperconectado, provocando una quiebra del monopolio del poder que poseían los medios de comunicación tradicionales y las élites a la hora de construir la realidad social y política (McNair, 2006), y facilitando el monitoreo en tiempo real de las audiencias, en sintonía con una opinión pública en continuo proceso de cambio (Anstead y O'Loughlin, 2015).

Las nuevas plataformas tecnológicas y audiovisuales, contribuyen a redefinir el papel clásico de los medios tradicionales en la conformación de la opinión pública (Lippmann, 1922), propiciando una convergencia (Jenkins, 2008) e hibridación mediática (Chadwik, 2013), en la que los nuevos medios conviven con los medios tradicionales, generando un consumo y formato multipantalla, que permite a los usuarios compartir su experiencia televisiva (Esteinou, 2017), a través de la formación de comunidades virtuales (Marzal y Zallo, 2016), abriendo además la posibilidad de generar un debate y conversación en tiempo real.

Con esta perspectiva, nos situamos ante un nuevo modelo televisivo, que entronca tanto con las teorías participativas de la audiencia (Livingstone, 2013; Carpentier, 2011), como con las teorías de 
la función social de la televisión como servicio de interés general y público, así como la defensa de una política mediática activa y fortalecedora de la relación entre emisor y receptor, aprovechando los nuevos recursos digitales (Tremblay, 2016; Trappel, 2016; Horowitz, 2015). Estas realidades paralelas de consumo multipantalla, entre audiencias tradicionales y sociales, ha generado el concepto de "audiencia social", bajo el cual se recogen el número de individuos que realizan alguna mención sobre un contenido televisivo en una red social determinada (Quintas y González, 2014).

Existen diversas investigaciones que analizan la actividad en redes sociales durante los debates electorales televisados, entre las cuales cabe destacar los trabajos de López-García (2016), Vergeer y Franses (2016), Trilling (2015) y D'heer y Verdegem (2015). Precisamente, una de las teorías más referidas en la literatura sobre debates políticos y redes sociales digitales es la Teoría de las Segundas Pantallas (Horning, 2017; De Zúñiga, García-Perdomo y McGregor, 2015), que analiza los factores que predicen la selección y visionado de información a través del empleo, único o combinado, de distintos positivos, desde la televisión, pasando por el teléfono, tableta u ordenador portátil.

Vaccari, Chadwick y O'Loughlin (2015) se refieren a la doble pantalla o segunda pantalla como el conjunto de prácticas que involucrar la integración entre los medios de transmisión en vivo y las redes sociales, es decir, la articulación entre la recepción del contenido de los medios tradicionales y la interacción generada en redes sociales con dichos contenidos. Sin embargo, tampoco existe un amplio consenso a la hora de referirse a este fenómeno, ante la variedad creciente de formatos y soportes, lo cual lleva a la consideración del término multipantalla.

En cualquier caso, este fenómeno se ha estudiado durante los últimos años, principalmente, alrededor de eventos de alta participación política, como es el caso de los debates electorales televisados (Gil de Zúñiga y Liu, 2017).

Además, la creciente popularidad de los teléfonos móviles y sus aplicaciones, ha generado una línea de investigación sobre cómo la comunicación móvil contribuye al compromiso democrático (Campbell y Kwak, 2011; Kim, Chen y Wang, 2016), en su utilización como segunda pantalla y pantalla única.

\subsection{La seducción de las segundas pantallas: gustar, compartir o algo más}

La cuestión y debate de fondo es si las redes sociales contribuyen a "ensanchar" el espacio público de la comunicación política -y en el caso que nos ocupa contribuyen al debate ciudadano sobre los debates electorales- dando más protagonismo a los ciudadanos (Wolton, 2007) o, por el contrario, actúan como caja de resonancia de los medios offline (López-Meri, 2016).

Y dentro de esta horquilla entran distintas consideraciones y grados, desde la acción simple de ver y leer, a otras como darle a "me gusta", siendo esta la forma de interactuar preferida por el público con los partidos políticos, pues exige menos esfuerzo y compromiso por parte del usuario (MartínezRolán, 2018). Los denominados "botones sociales" reflejan, según algunos autores, estados afectivos cortos (Gerlitz y Helmond, 2013) y débiles (Guy et al., 2016) y un compromiso de bajo coste e implicación- a comentar o responder y compartir una publicación- (Barger y Labrecque, 2013) e incluso, a encontrar, escuchar y movilizar a una comunidad alrededor de una cuestión (BallesterosHerencia, 2019).

Ciertamente, hay herramientas para la medición para comprobar las interacciones generadas, a través de las redes sociales, que inducen a la conversación, siguiendo algunos de los principios dialógicos 
básicos (información útil, generación de visitas reiteradas y mantenimiento del bucle dialógico), establecidos por Kent y Taylor (1998) y adaptados por Ribalko y Seltzer (2010).

De igual modo, existe igualmente un debate académico alrededor de la definición del engagement político (índice de respuesta de los usuarios sobre los comentarios realizados a través de las redes sociales) y la participación, a partir de la consideración del mismo como la interacción de una audiencia con un contenido digital, y del propósito básico de medir el compromiso del ciudadano con una organización o partido e, incluso, su implicación emocional, tal y como sostienen Pedersen et al. (2014).

Pero como también apunta González (2013), las interacciones en la red social no son únicamente las veces que se comparte el contenido en el perfil de un usuario, sino también la cantidad de veces que esos contenidos se comentan, ganan un 'me gusta' o lo comparten otras personas. Comentar y compartir un mensaje supone, por tanto, una participación más activa, que revela una concordancia con lo expresado en el mensaje original y que, además, contribuye a darle una mayor difusión (Valerio et al., 2015).

Precisamente, tratar de averiguar la implicación de los usuarios y su comportamiento político a través de la utilización de las segundas pantallas es uno de los propósitos del análisis de las segundas evaluaciones, a través del modelo de Orientación-Estímulo-Razonamiento-Orientación-Respuesta (OSROM), que se observa como esencial para valorar no sólo el proceso de búsqueda de información, sino también el procesamiento, razonamiento, orientación y posibles efectos de la misma (Hsuan-Tin, 2019) y, en definitiva, su nivel de contribución activa a la conversación.

Una vez descartado el ruido informativo generado por la intoxicación de las redes sociales (bots, trolls, etc.) y de prácticas como el "clicktivismo" (Shulman, 2009), que busca incrementar de forma inducida el impacto de los mensajes, nos encontramos con acciones que pueden ir desde el simple "slacktivismo" o activismo de salón y holgazán (Christensen, 2011), a la utilización de las nuevas tecnologías como herramientas para la canalización del malestar social y las protestas ciudadanas, que revelan el ejercicio del poder de la sociedad civil a través de las redes sociales (Caldevilla, Rodríguez y Barrientos, 2019).

De hecho, tal y como señalan Dader y Campos (2017), en comunicación política resulta muy habitual la utilización, durante las campañas electorales, de voluntarios o profesionales, para difundir de forma masiva información durante la celebración de los debates electorales y demás eventos destacados de los partidos, en muchas ocasiones mediante la utilización intensiva de hashtags como una estrategia de marca política (Mas y Guerrero, 2019).

Todo ello teniendo en cuenta la tendencia de los usuarios de las redes sociales a la homofilia, es decir, a relacionarse entre iguales, sin posibilidad de abrirse a lo que consideran diferente (McPherson, Smith y Cook, 2001), a través del establecimiento de burbujas (Davies, 2018) de información y cámaras de resonancia (Del Vicario et al., 2018), que provocan una fuerte polarización de las opiniones.

En definitiva, el debate sobre las posibilidades de conversación y debate político en las redes sociales se mueve entre quienes sostienen que las redes introducen ruido y distracción -provocando incluso un menor recuerdo y comprensión, socavando la capacidad de la audiencia para deliberar y discutir (Gorkovenko y Taylor, 2019- y quienes indican que se trata de un complemento y segunda valoración, voluntaria e intencional, que contribuye a incrementar las posibilidades de atención y 
persuasión en las campañas electorales, incrementando en consecuencia el compromiso cognitivo y conductual (Chadwick, O'Loughlin y Vaccari, 2017).

\subsection{Agenda temática}

Tradicionalmente se ha entendido la interacción entre los medios de comunicación y la ciudadanía como un proceso en el que los primeros logran posicionar ciertos temas de su agenda entre las prioridades de los segundos, tal y como establece la teoría de agenda-setting (McCombs y Shaw, 1972). A partir de esta teoría, cabe preguntarse si, de la misma manera, las problemáticas de la ciudadanía pasan a ser temas de interés para los actores mediáticos y políticos y viceversa.

El énfasis que los medios ponen sobre los temas en la cobertura de una campaña electoral influye sobre la selección, priorización, enfoque y encuadre de los mismos, de acuerdo con las teorías del priming (Iyengar y Kinder, 1987) y framing (Reese, Gandy y Grant, 2001).

Igualmente, los debates electorales son un proceso que nos permite observar cuáles son los temas en las agendas y temas de campaña de los medios, los políticos y los ciudadanos, así como la posible existencia de una intersección y correlación temática.

El análisis de los debates nos permite abordar de forma conjunta tanto la agenda mediática, como la agenda política, y analizar el posible seguimiento e impacto de ambas por los ciudadanos, teniendo en cuenta el papel de la prensa, primero, en la jerarquización y establecimiento de los temas en la fase de precampaña $\mathrm{y}$, seguidamente, durante la campaña, por parte de la televisión, en la enfatización de los mismos (López-López, Puentes-Rivera y Rúas-Araújo, 2017).

En este sentido, es de especial relevancia conocer en qué medida los ciudadanos presentan agendas o encuadres diferentes y, en concreto, para el caso que nos ocupa, si los temas de interés de los ciudadanos que siguieron los debates a través de los medios tradicionales (prensa, radio y TV) coinciden con los temas considerados como prioritarios por los usuarios de las redes sociales.

Precisamente, algunas de las evidencias sobre la existencia de un claro ensamblaje entre las agendas mediática y política a la hora de estructurar los temas de discusión en los debates televisados (RúasAraújo y Mazaira-Castro, 2019), invitan al seguimiento y análisis de los temas y asuntos de interés de los usuarios de las redes sociales, para comprobar las posibles semejanzas o diferencias entre las audiencias tradicionales y las nuevas audiencias.

\section{Metodología}

Esta investigación se realizó de acuerdo con los siguientes objetivos:

- OG1: Identificar los diferentes públicos que emplean las redes sociales para obtener o ampliar información acerca de los debates televisados del 22 y 23 de abril de 2019.

- OG2: Observar y comparar los asuntos que más interés suscitaron entre las audiencias de los nuevos medios y los medios tradicionales.

- OG3: Detectar si los internautas participaron a través de comentarios y mensajes compartidos, así como el momento en que lo hicieron, antes, durante o después de la celebración del debate televisado.

- OG4: Conocer el grado de credibilidad que los usuarios de redes sociales otorgaron a los datos ofrecidos por los candidatos en los debates televisados. 
Para ello, se realizó una encuesta para conocer cuáles son las pautas de consumo en las redes sociales durante los debates electorales televisados emitidos por RTVE y el grupo Atresmedia durante las jornadas del 22 y 23 de abril respectivamente, con motivo de las elecciones generales de abril de 2019.

Para desarrollar la presente investigación, se empleó una metodología cuantitativa, a partir de la realización de una encuesta telefónica, con una muestra aleatoria simple sobre la población general del área elegida. Esta encuesta telefónica está compuesta por 22 preguntas, que se plantearon y procesaron a partir de la jornada posterior al segundo debate (24 de abril).

Se llevaron a cabo más de 8.000 llamadas telefónicas en el área de las ciudades de Madrid, Barcelona, A Coruña y Vigo, abarcando una población total de 6.204 .000 personas, de las que se obtuvieron un total de 734 respuestas completas, con un margen de error sobre el total de la muestra inferior al $4 \%$.

Tras la recogida de las respuestas de los encuestados, se analizan los datos extraídos de la muestra con el fin de establecer una tendencia en el impacto de los debates electorales televisados a través de los medios tradicionales y los nuevos medios, además de describir el perfil de estas audiencias.

\section{Resultados}

\subsection{Quiénes han visto los debates electorales}

Un 52,2\% de los encuestados visualizaron alguno de los dos debates emitidos por RTVE y el grupo Atresmedia (22 y 23 de abril), alcanzando resultados similares para ambos canales

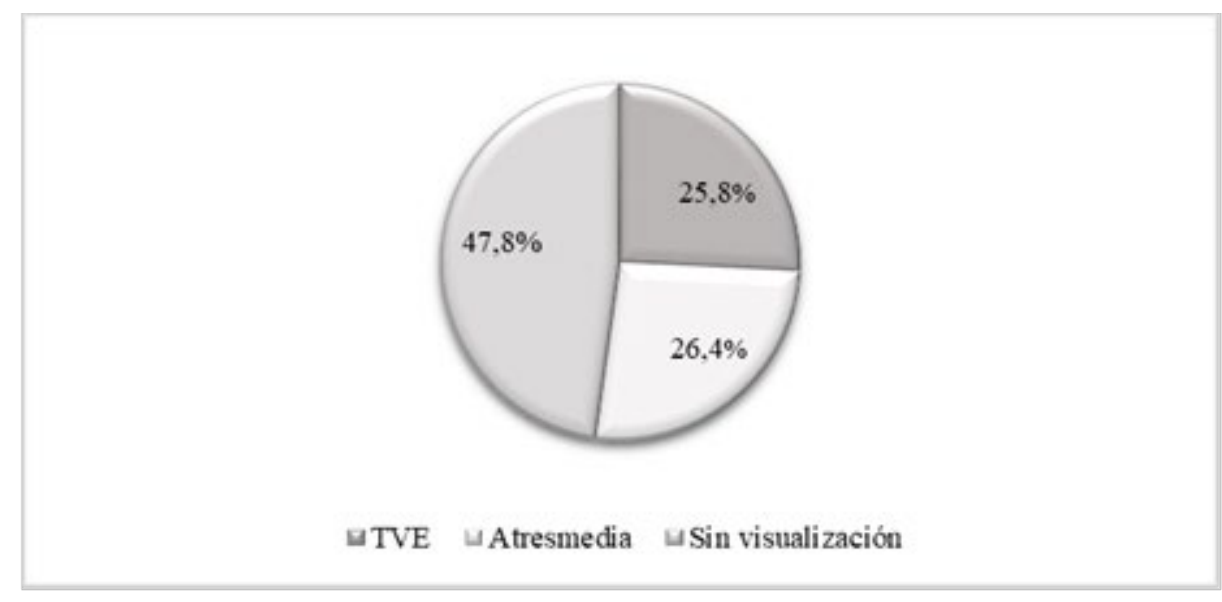

\section{Gráfico 1. Visualización debate. \\ Fuente: elaboración propia.}

Del total de quienes siguieron el primer debate (RTVE, 25,8\%) un 24,9\% corresponde a Madrid, $23,9 \%$ a Barcelona, A Coruña 26,8\% y Vigo $28,3 \%$. Por sexo, un $28,5 \%$ son hombres y un $23,3 \%$ mujeres y por rango de edad, un $21,1 \%$ son menores de 35 años, el $29,3 \%$ tienen entre 35 - 55 años y un $26,8 \%$ mayores de 55 años.

De los datos obtenidos del segundo debate emitido por el grupo Atresmedia, pertenecen a Madrid el $26,6 \%$, Barcelona $25,7 \%$, A Coruña $28,9 \%$ y Vigo $25,3 \%$. Por sexo un $29,75 \%$ es público masculino 
y un $23,3 \%$ femenino; mientras que por rango de edad un $29,7 \%$ pertenece a la categoría de menores de 35 años; un 29,3\%, entre 35 - 55 años y un 27,9\% son mayores de 55 años.

\subsection{Qué temas interesan más}

En cuanto a los temas, economía y pensiones son los asuntos que más interés suscitaron entre los encuestados (en torno al 16\% y 15\%) seguido en un tercer nivel de importancia por el paro (casi un $12 \%)$, así como políticas sociales $(9 \%)$. En materia de política territorial, la independencia de Cataluña o temas de índole social como inmigración o violencia de género, desciende considerablemente el número de encuestados que le otorgan mayor atención.

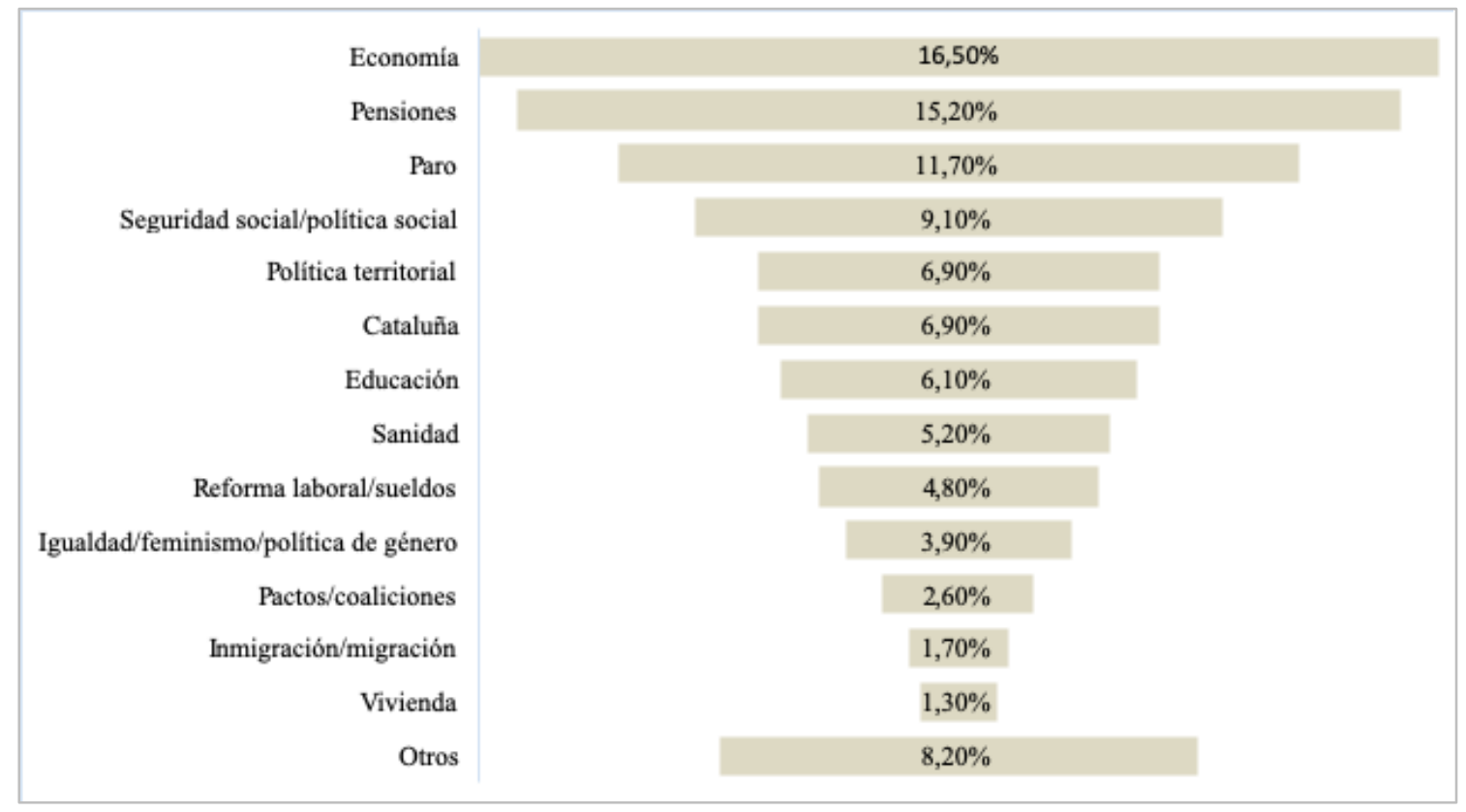

Gráfico 2. Interés de las audiencias en los temas del debate televisado.

Fuente: elaboración propia.

\subsection{Quiénes consultan información sobre los debates televisados en otros medios}

De quienes vieron ambos debates televisados, tan solo un $27,8 \%$ obtiene más información acerca de los mismos a través de otros medios de comunicación (prensa papel/internet, radio, redes sociales $\mathrm{u}$ otras webs) siendo mayoritariamente personas de entre 35 y 55 años las que más realizaron esta consulta. Como medio de consulta, las diferentes redes sociales se sitúan en cabeza, con un $21 \%$ seguidos por la prensa a través de internet y la prensa en papel. 


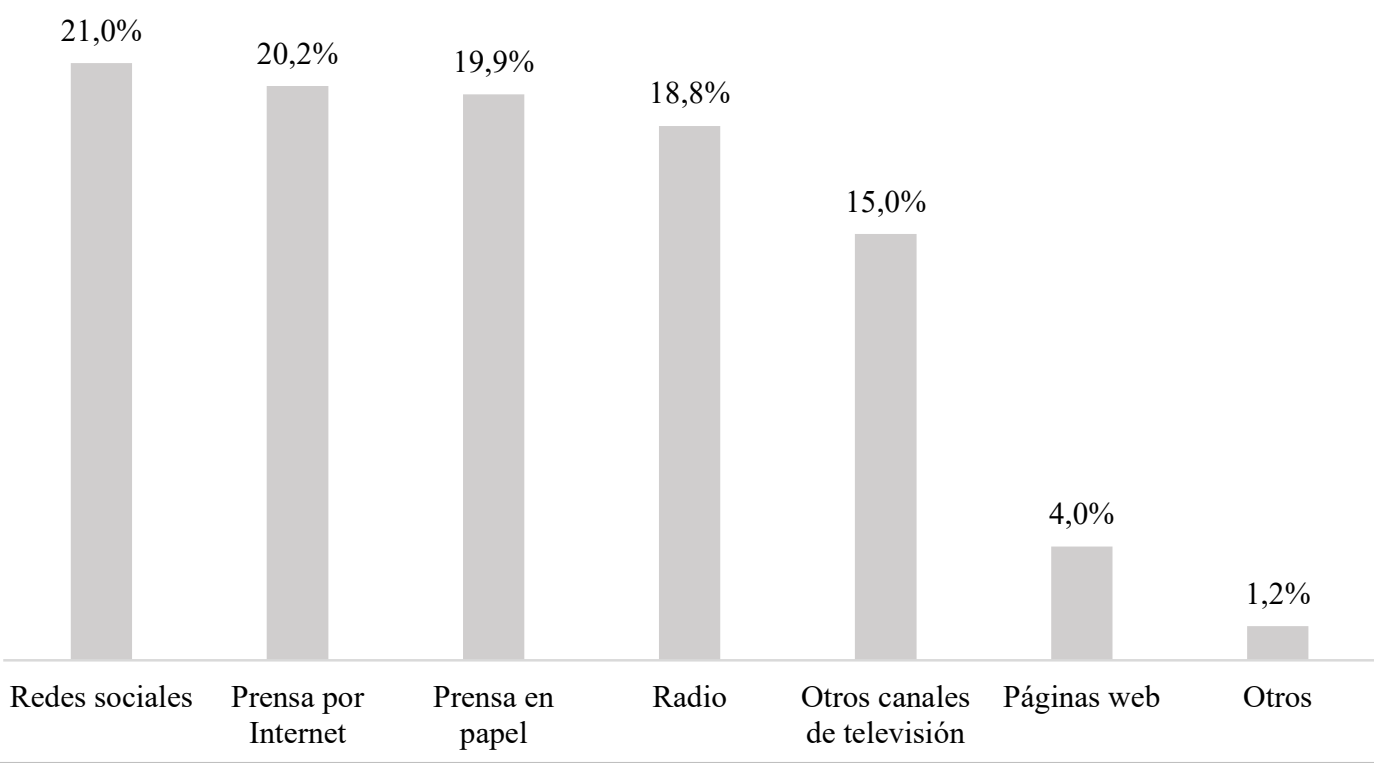

Gráfico 3. Consulta adicional de información acerca de los debates televisados.

Fuente: elaboración propia.

\subsubsection{Los debates televisados en las redes sociales}

Del conjunto de personas que aseguran informarse sobre alguno de los dos debates televisados del 22 y 23 de abril a través de las redes sociales, Facebook se sitúa como la red favorita como medio de consulta, seguido de Twitter, con diferencias significativas con las demás redes sociales (Instagram, WhatsApp y YouTube,) que juegan un papel secundario y tan solo consiguen un $32 \%$ de audiencia conjunta, algo que contrasta con la tendencia actual de crecimiento de estas redes.

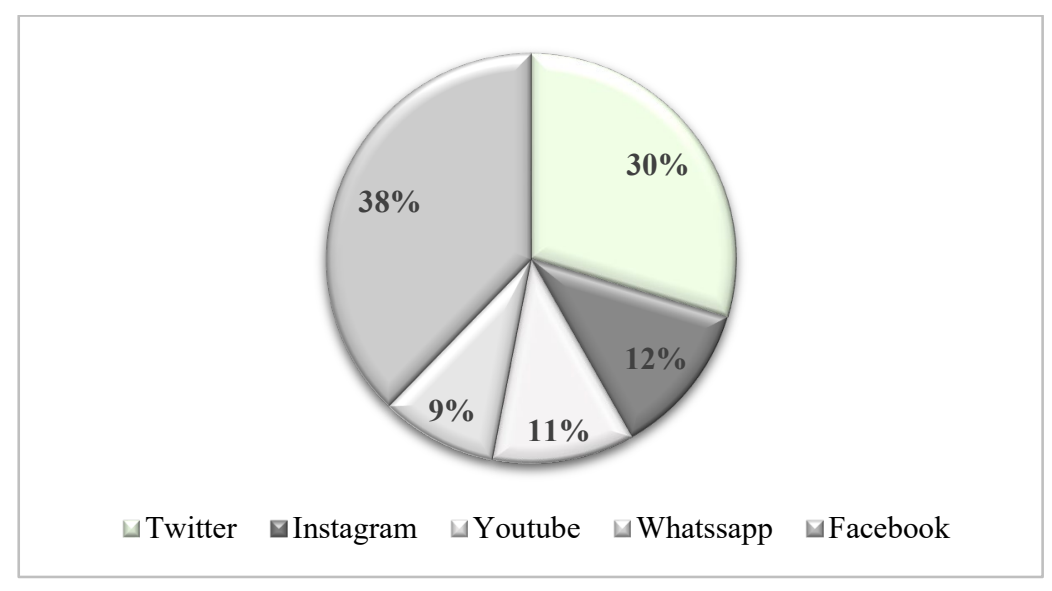

Gráfico 4. Otros medios de consulta.

Fuente: elaboración propia.

En cuanto al perfil de usuarios que emplean las redes como medio de información de los debates televisados, se extraen los siguientes resultados a partir de variables sociodemográficas: las mujeres prestan más atención que los hombres (casi un $21 \%$ público femenino frente al $16 \%$ masculino, 
siendo Facebook y Twitter las que captan mayor interés de los internautas $(9 \%$ y 5,6\% para Facebook, y 5,8\% y 5,6\% para Twitter).

Por edad, los menores de 35 son los que más las emplean como medio de información (un 45\%), siendo Twitter quien cobra mayor importancia (15\%) seguido de Facebook (11,7\%). Las redes sociales tienen un papel secundario entre los usuarios de más de 35 años quienes prefieren otros medios como la prensa en papel e internet, ocupando en torno a un 16\% de la atención sin distinción de red.

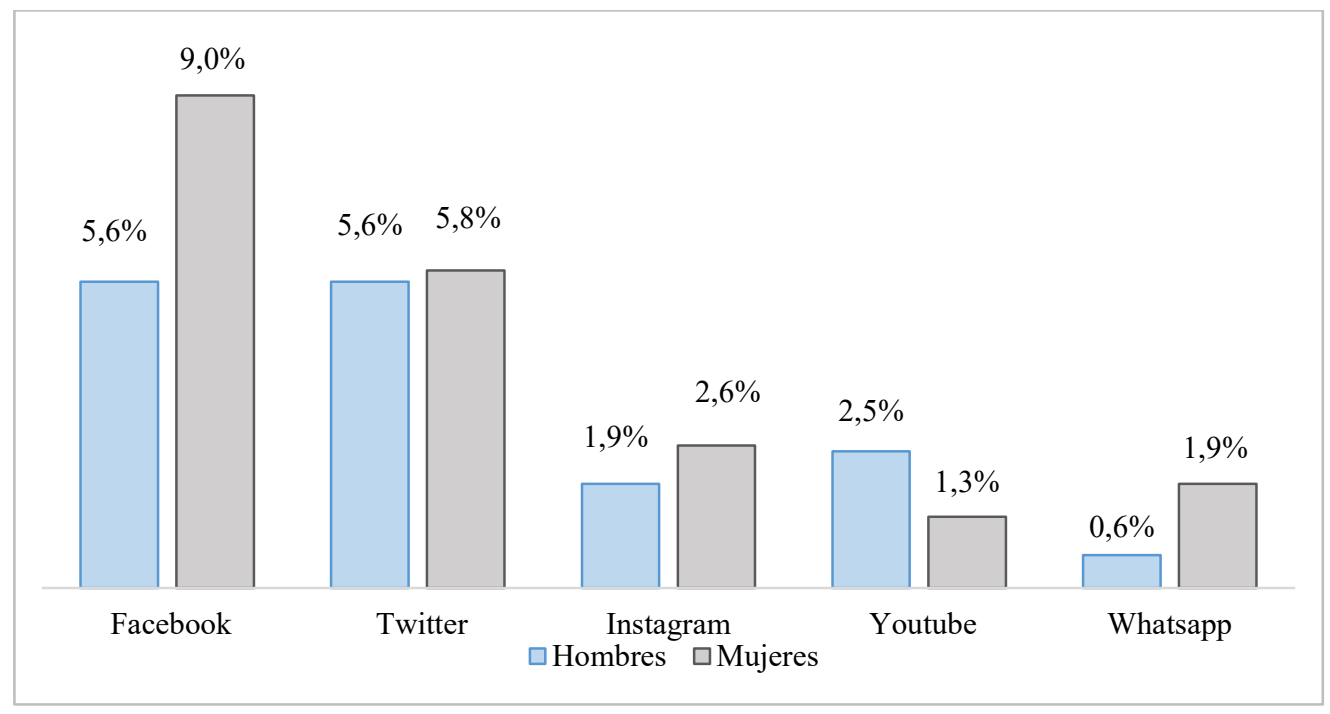

Gráfico 5. Uso de redes sociales sobre los debates electorales.

Fuente: elaboración propia.

En cuanto a participación de los internautas, tan solo un $43 \%$ de los encuestados forman parte del núcleo de participación activa, configurándose este grupo por personas entre 35 y 55 años, sin distinción entre hombres y mujeres.

Con respecto a la frecuencia de consulta y/o participación, más de la mitad de los encuestados (55\%) reconoce haber interactuado a través de las redes sociales con contenidos relacionados con ambos debates, estando muy equiparadas las reacciones, tanto antes como durante la realización de los mismos.

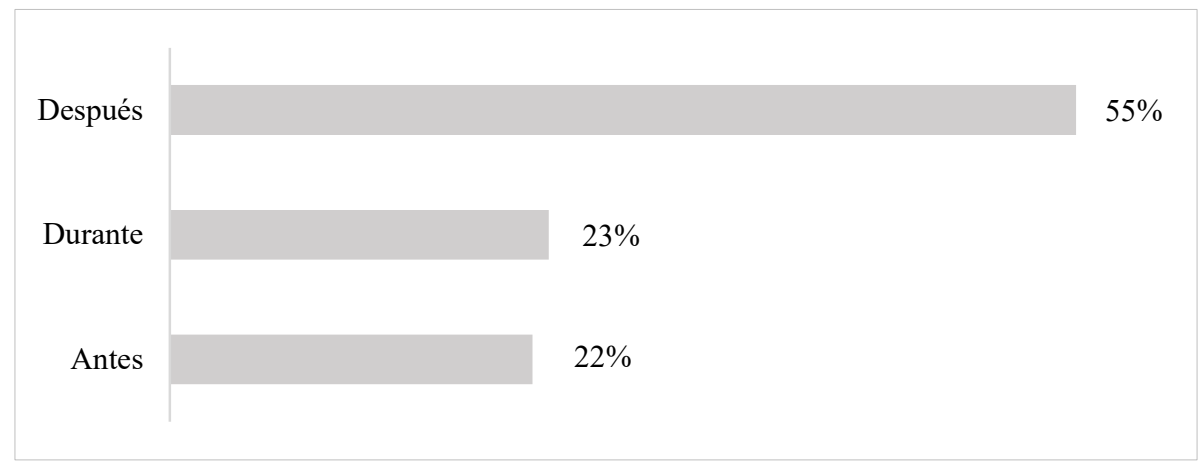

Gráfico 6. Consulta del debate.

Fuente: elaboración propia. 
Otro propósito de esta investigación es observar si existen coincidencias entre los temas que han interesado más a los de las redes sociales con respecto a las audiencias de medios tradicionales que han seguido los debates. Estos resultados se pueden observar en la gráfica 2 (economía, pensiones y paro). Como pauta general, los internautas continúan con los mismos criterios de selección que en el caso de los usuarios de medios tradicionales, pero lo hacen en diferente grado de importancia: lo referido a asuntos económicos ocupa un $18,6 \%$, mientras que pensiones y paro descienden a un $12,70 \%$ y $11 \%$ respectivamente. Los demás asuntos tratados continúan el mismo orden de jerarquía en ambos casos y con valores similares.

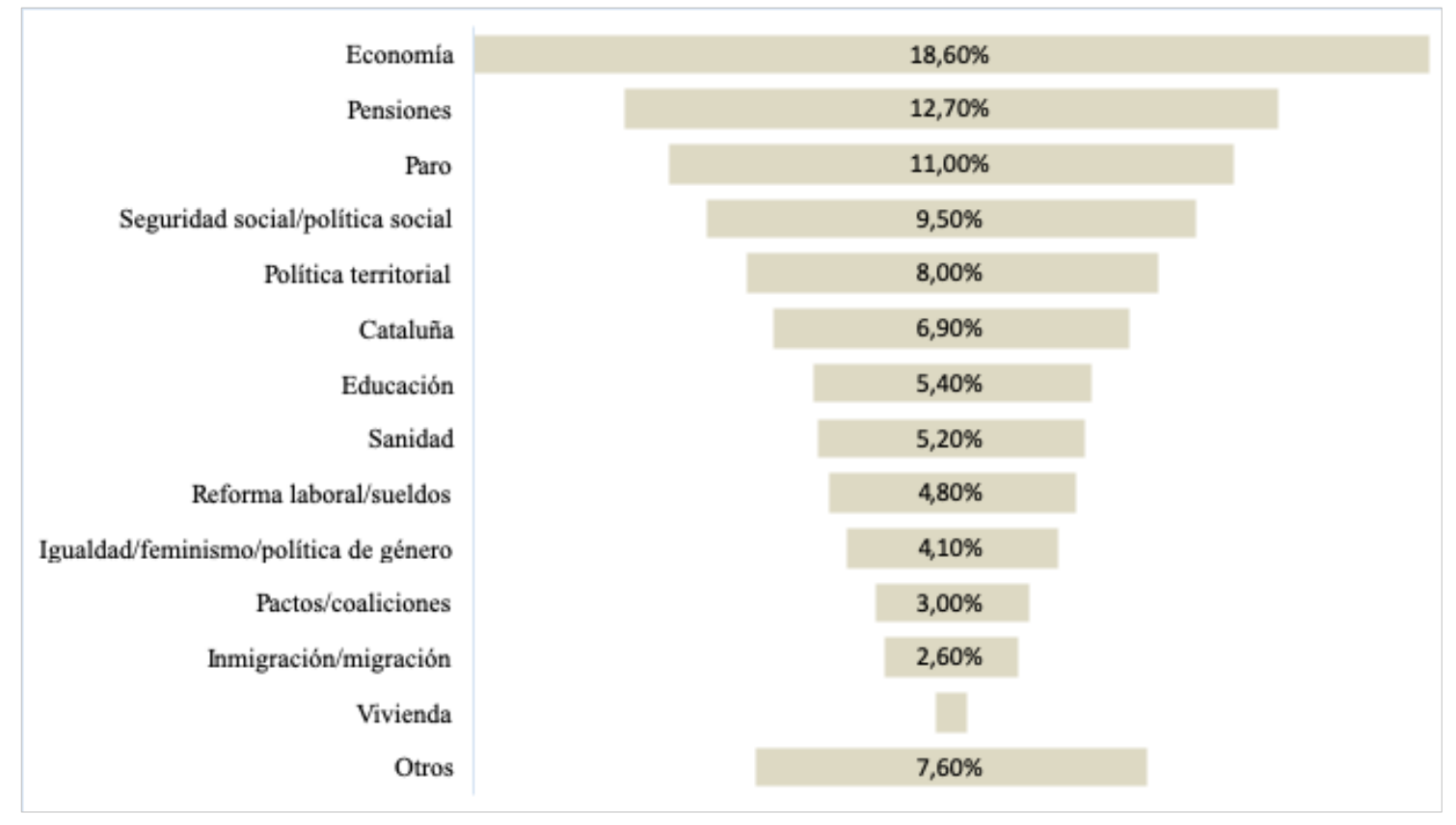

Gráfico 7. Interés temático en redes sociales.

Fuente: elaboración propia.

Por otro lado, las audiencias de las redes sociales otorgan poca credibilidad a los datos y cifras indicados durante el debate por los candidatos (gráfico 8).

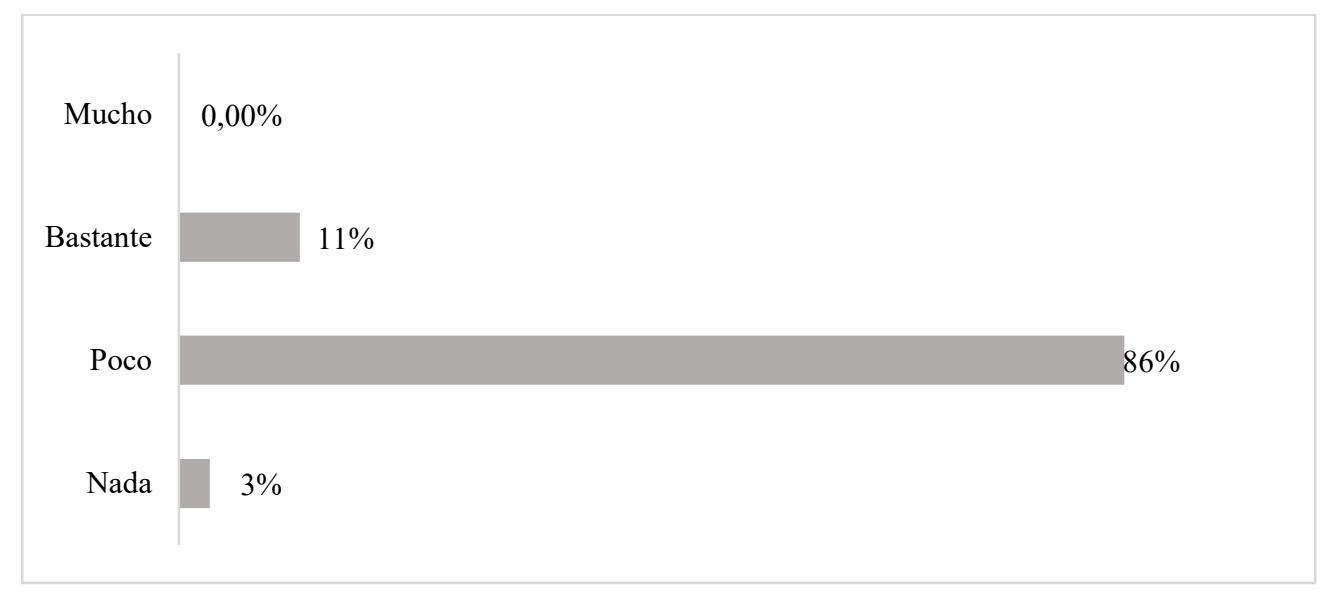

Gráfico 8. Credibilidad de datos en redes sociales.

Fuente: elaboración propia. 


\section{Discusión y conclusiones}

Los resultados de la encuesta realizada en esta investigación muestran que en lo referido al primer objetivo específico, el consumo de los debates televisados a través de redes sociales se aprecia que existe una brecha de edad y género en el consumo de información sobre los debates televisados a través de redes sociales: las mujeres son más activas que los hombres, mientras que los jóvenes se alejan de los medios de comunicación tradicionales para interesarse por lo que sucede en torno a los debates electorales, dando lugar a perfiles proactivos en la red.

Por otro lado, Facebook y Twitter poseen mayor interés por parte de los usuarios para acceder a la información de los debates televisados, a pesar de que estas han sufrido un parón en su crecimiento en comparación con Instagram, YouTube y WhatsApp. Los internautas aprovechan su fortaleza principal: información en tiempo real del propio debate televisado y en la que la escucha activa de otros usuarios (sobre todo en Twitter) hacen posible el intercambio de información.

A pesar de que Instagram ha duplicado el número de usuarios registrados a un ritmo vertiginoso, no entra dentro de las preferencias de los usuarios a la hora de informarse sobre temas de actualidad, como son los debates electorales televisados. Esto se debe a que se considera una red en la que prima la imagen a favor de las marcas y, además, no permite generar tráfico en su "timeline" a través de sus publicaciones y redirigir a los usuarios hacia otros perfiles, ya sean de las propias cadenas que emiten el debate o hacia el de los candidatos políticos. Esta oportunidad en cambio sí es posible a través de Facebook y Twitter, esta última red -normalmente abierta- permite además acceder a las publicaciones de los denominados "influencers" políticos, personas con cierta notoriedad reconocida en el ámbito y generar hilos de conversación a los que los internautas generan confianza.

En el caso de WhatsApp los bajos resultados generados se deben a que es una red considerada todavía de mensajería instantánea entre los propios contactos y, por lo tanto, de carácter personal y privada, por lo que el acceso a contenidos relativos al debate televisado únicamente gira en torno a lo que estos círculos más cercanos comparten entre el propio universo de contactos de la persona.

Con respecto a la red social YouTube, aunque ésta ofrece funcionalidades sociales a través de las que adquirir mayor información acerca de los debates televisados y sobre todo tras la realización de los mismos -es una plataforma que actúa como repositorio de las mismas cadenas para visualizar el debate completo- no logra posicionarse dentro de las predilectas de las audiencias en el área estudiada, más que para viralizar momentos o comentarios más anecdóticos del propio debate electoral, como pueden ser la frase de "Ya acabó usted de mentir? Ahora me toca a mí" de Albert Rivera a Pedro Sánchez, el intercambio de libros entre ambos candidatos, o el pergamino en el que Albert Rivera detalla a Pedro Sánchez todos los casos de ERE de Andalucía.

En lo referido al segundo de los objetivos (OG2), tanto las audiencias de redes sociales como de medios tradicionales muestran su principal preocupación hacia asuntos de índole económica, paro y pensiones. Relacionando los datos sociodemográficos de los encuestados usuarios de redes con los intereses que los mismos manifiestan en torno al debate, se constata que el colectivo de jóvenes y mediana edad son los que muestran mayor desafección con el actual panorama económico y el crecimiento del paro, aunque prestan menos interés en torno a otros asuntos directamente ligado a estos, como son la reforma laboral o los sueldos.

En lo que respecta al tercer objetivo OG3, se aprecian, claramente, diferencias entre los usuarios con una participación pasiva (que se nutren de información, o bien buscan expresamente contenidos relacionados, pero en ningún momento los generan) y quienes muestran una participación activa, en 
la que comparten y comentan información. Por lo tanto, estamos ante una participación lineal, con escaso feedback, en la que prevalece el "mirar" más que comentar, compartir, o dar un "me gusta", fundamentalmente porque estas acciones son consideradas como un modo de mostrar al público cercano el posicionamiento político de la persona.

En cuanto al último objetivo de investigación (OG4) relativo a conocer el grado de credibilidad que los usuarios de redes sociales otorgaron a los datos ofrecidos por los candidatos en los debates televisados, las cifras ponen de manifiesto la desconfianza total de las audiencias hacia los líderes de los partidos, así como en la política general española. Por otro lado, queda patente que la información ofrecida en los debates electorales televisados no influye sobre los votantes ni es determinante a la hora de movilizar a los electores hacia el voto de una u otra fuerza política en las urnas. Además, los datos ofrecidos en los debates televisados no tienen por qué permanecer en el recuerdo de las audiencias, mientras que, por el contrario, una publicación que se vuelve viral en redes sociales (ya sea vídeo, meme, o tuit) tiende a ser más recordada e influir en el voto indeciso.

En cualquier caso, al margen de la medición de las audiencias, queda también para el debate la cuestión sobre si el segundo "cribado" de información, derivado de la utilización de las segundas pantallas, influye en la participación política y la decisión del voto, en la línea de lo expresado por los autores e investigaciones señalados al principio de esta investigación.

\section{Referencias bibliográficas}

Anstead, N. \& O'Loughlin, B. (2015). Social Media Analysis and Public Opinion: The 2010 Uk General Election. Journal of Computer-Mediated Communication, (20), 204-220.

Ballesteros-Herencia, C. A. (2019). La representación digital del engagement: hacia una percepción del compromiso a través de acciones simbólicas. Revista de Comunicación, 18(1), 215-233.

Barger, V. A. \& Labrecque, L. (2013). An Integrated Marketing Communications Perspective on Social Media Metrics. International Journal of Integrated Marketing Communications, 64-76.

Berrocal, S. (2005). La información política en televisión: ¿apatía o interés entre los telespectadores? Comunicar, 25(5).

Campbell, S. W. \& Kwak, N. (2011). Political involvement in "mobilized" society: The interactive relationships among mobile communication, network characteristics and political participation. Journal of Communication, (61), 1005-1024.

Caldevilla, D., Rodríguez, J. y Barrientos, A. (2019). El malestar social a través de las nuevas tecnologías: Twitter como herramienta política. Revista Latina de Comunicación Social, (74), 1264-1290.

Carpentier, N. (2011). Managing Audience Participation: The Construction of Participation in an Audience Discussion Programme. European Journal of Communication, 16(2), 209-232.

Castells, M. (2009). Comunicación y poder. Alianza.

Chadwick, A., O'Loughlin, B. \& Vaccari, C. (2017). Why people dual screen political debates and why it matters for democratic engagement". Journal of Broadcasting \& Electronic Media, (61), 220-239. 
Chadwick, A. (2013). The Hybrid Media System, Politics and Power. Oxford University.

Christensen, H. S. (2011). Political activities on the Internet: Slacktivism or political participation by other means? First Monday, 16(2).

Dader, J. L. y Campos, E. (Coords.) (2017). La Búsqueda Digital del Voto. Cibercampañas electorales en España, 2015-16. Tirant lo Blanch.

Del Vicario, M., Quattrociocchi, W., Scala, A. \& Zollo, F. (2018). Polarization and Fake News: Early Warning of Potential Misinformation Targets. ARXIV.

D'heer, E. \& Verdegem, P. (2015). What social media data mean for audience studies: multidimensional investigation of Twitter use during a current affairs TV programme. Information, Communication \& Society, 18(2), 221-234.

Esteinou, J. (2017). Los medios electrónicos. Anuario de Investigación de la Comunicación. CONEICC, (24), 31-52.

Gallego, M. y Bernárdez, A. (2017). Influencia y repercusión mediática de los debates "cara a cara" celebrados ante las elecciones generales de 2008 en España: José Luis Rodríguez Zapatero (PSOE) vs Mariano Rajoy (PP). Vivat Academia. Revista de Comunicación, (141), 139-154.

Gil de Zúñiga, H. \& Liu, J. H. (2017). Second screening politics in the social media sphere: Advancing research on dual screen use in political communication with evidence from 20 countries. Journal of Broadcasting \& Electronic Media, 61(2), 193-219.

Gil de Zúñiga, H., García-Perdomo, V. \& McGregor, S. (2015). What is Second Screening? Exploring Motivations of Second Screen Use and Its Effects on Online Political Participation. Journal of Communication, (65), 793-815.

Gorkovenko, K. \& Taylor, N. (2019). Audience and Expert Perspectives on Second Screen Engagement with Political Debates. ACM International Conference on Interactive Experiences for TV and Online Video (TVX '19), Salford (Manchescher): United Kingdom.

Guy, I., Ronen, I., Zwerdling, N., Zuyev-Grabovitch, I. \& Jacovi, M. (2016). What is your organization 'like'? A study of liking activity in the enterprise. In CHI 16 Proceedings of the 2016 CHI Conference on Human Factors in Computing Systems. Newy York: ACM (Association for Computing Machinery).

Horowitz, M. A. (2015). Public Service Media and Challegue of Crossing Borders: Assessing New models. Medijske Studije, 6(12), 80-90.

Hsuan-Ting, C. (2019). Second Screening and the Engaged Public: The Role of Second Screening for News and Political Expression in an OSROR Model. Journalism \& Mass Communication Quarterly, 1-21.

Huertas Bailén, A. (2002). La audiencia investigada. Editorial Gedisa.

Iyengar, S. \& Kinder, D. (1987). News That Matters. University of Chicago Press. 
Kim, Y., Chen, H. T. \& Wang, Y. (2016). Living in the smartphone age: Examining the conditional indirect effects of mobile phone use on political participation. Journal of Broadcasting \& Electronic Media, (60), 694-713.

Kent, M. L. \& Taylor, M. (1998). Building dialogic relationships through the World Wide Web. Public Relations Review, 24(3), 321-334.

Lippmann, W. (1922). Public Opinion. Macmillan.

Livingstone, S. (2013). The Participation Paradigm in Audience Research. The Communication Review, (16), 21-30.

López-López, P. C.; Puentes-Rivera, I. y Rúas-Araújo, J. (2017). La comunicación política en las elecciones gallegas del 25 de septiembre de 2016: la agenda temática en el debate de la TVG. En: Herrero-Gutiérrez, F. J.; Mateos-Martín, C.; Toledano-Buendía, S.; Ardèvol-Abreu, A.; Trenta, M. Del verbo al bit (Cuadernos Artesanos de Comunicación). Universidad de La Laguna, 1431-1460.

López-García, G. (2016). 'Nuevos' y 'viejos' liderazgos: la campaña de las elecciones generales españolas de 2015 en Twitter. Communication \& Society, 29(3), 149-167.

López-Meri, A. (2016). Periodismo en Twitter. La contribución de los usuarios al flujo informativo. Cuadernos.info, (39), 241-257.

Martínez Rolan, X. (2018). La actividad de los partidos políticos españoles en Facebook 20142018. La tiranía del algoritmo. Revista de Comunicación de la SEECI, (47), 143-155.

McCombs, M. \& Shaw, D. (1972). The agenda setting function of mass media. Public Opinion Quarterly, (36).

Mas, L. \& Guerrero, F. (2019). The use of hashtags as a political branding strategy. Revista Internacional de Relaciones Públicas, 17, 5-24.

Marzal, J. \& Zallo, R. (2016). Las televisiones públicas de proximidad ante los retos de la sociedad digital. Communication \& Society, 29(4), 1-7.

McNair, B. (2006). Cultural Chaos: Journalism and Power in a Globalised World. Routledge.

McPherson, M., Smith, L \& Cook, J. M. (2001). Birds of a Feather: Homophily in Social Networks. Annual Review of Sociology, (27), 415-444.

Pedersen, S., Baxter, G., Burnett, S., Goker, A., Corney, D. \& Martin, C. (2014). Backchannel chat: Peaks and troughs in a Twitter response to three televised debates during the Scottish Independence Referendum Campaign 2014. Aberdeen Business School Working Paper Series, $7(2), 1-33$.

Quintas, N. y González, A. (2014). Audiencias activas: Participación de la audiencia social en la televisión. Comunicar, 43(22), 83-90.

Reese, S., Gandy, O. \& Grant, J. (2001). Framing Public Life: Perspectives on Media and Our Understanding of the Social World. Routledge. 
Ribalko S. \& Seltzer, T. (2010). Dialogic communication in 140 characters or less: How Fortune 500 companies engage stakeholders using Twitter. Public Relations Review, 36(4), 336-341.

Rúas-Araújo, J. y Mazaira-Castro, A. (2019). Agenda mediática y política: ¿Amistades peligrosas? Un análisis desde el fact-checking. En: Conde-Vázquez, E.; Fontenla-Pedreira, J. y Rúas-Araújo, J. Debates Electorales Televisados: del antes al después, (151-172). Cuadernos Artesanos de Comunicación, (154).

Shulman, S. W. (2009). The case against mass e-mails: Perverse incentives and low quality public participation in US federal rulemaking. Policy \& Internet, (1), 23-53.

Téllez, N, Muñiz, C. y Ramírez, J. (2010). Función discursiva en los debates televisados. Un estudio transcultural de los debates políticos en México, España y los Estados Unidos. Palabra Clave, 13(2).

Trappel, J. (2016). Taking the public service remit forward across the digital boundary. International Journal of Digital Television, 7(3), 273-295.

Tremblay, G. (2016). Public Service Media in the Age of Digital Networks. Canadian Journal of Communication, 41(4), 191-206.

Trilling, D. (2015). Two different debates? Investigating the relationship between a political debate on TV and simultaneous comments on Twitter. Social Science Computer Review, 33(3), 259-276.

Vaccari, C., Chadwick, A. \& O’Loughlin, B. (2015). Dual Screening the Political: Media Events, Social Media, and Citizen Engagement. Journal of Communication, 65(6), 1041-1061.

Valerio, G., Herrera, D. J., Villanueva, F., Herrera, N. \& Rodríguez, M. C. (2015). The relationship between post formats and digital engagement: A study of the Facebook pages of Mexican universities. RUSC, 12(1), 50-63.

Vergeer, M. \& Franses, P. H. (2016). Live audience responses to live televised election debates: time series analysis of issue salience and party salience on audience behavior. Information, Communication \& Society, 19(10), 1390-1410.

Webster, J. G., Phalen, P. F. \& Lichty, L. W. (2014). Rating Analysis: Audience Measurement and Analytics. Routledge.

Wolton, D. (1998). El nuevo espacio público. Gedisa.

\section{AUTORES:}

\section{Julia Fontenla-Pedreira}

Licenciada en Periodismo por la Universidad de Santiago de Compostela, y Máster en Lengua y Comunicación en los Negocios por la Universidad de Vigo. Investigador predoctoral en formación FPI vinculada al proyecto de I+D+I (Retos) "DEBATv, Debates Electorales Televisados en España: Modelos, Proceso, Diagnostico y Propuesta" (Ref. CSO2017-83159-R), financiado por el Ministerio de Economía, Industria y Competitividad (MINECO), la Agencia Estatal de Investigación (AEI) y el Fondo Europeo de Desarrollo Regional (FEDER) de la Unión Europea (UE).

julia.fontenla.pedreira@uvigo.es

Orcid ID: https://orcid.org/0000-0001-8770-4761 


\section{José Rúas-Araújo}

Profesor titular de Comunicación Electoral e Institucional en la Facultad de Ciencias Sociales y de la Comunicación de la Universidad de Vigo y Director del Departamento de Comunicación Audiovisual y Publicidad de la misma institución. Es Investigador Principal de "DEBATv, Debates Electorales Televisados en España: Modelos, Proceso, Diagnostico y Propuesta" (CSO2017-83159$\mathrm{R}$ ), proyecto de $\mathrm{I}+\mathrm{D}+\mathrm{I}$ (Retos) del Ministerio de Ciencia, Innovación y Universidades. Sus líneas de investigación son la comunicación política y las neurociencias aplicadas a la comunicación persuasiva.

joseruas@uvigo.es

Índice H: 8

Orcid ID: http://orcid.org/0000-0002-1922-9644

Google Scholar: http://scholar.google.es/citations?user=DLiqzqkAAAAJ\&hl=es

\section{Erica Conde-Vázquez}

Graduada en Publicidad y Relaciones Publicas en la Universidad de Vigo, Máster en Marketing, Consultoría y comunicación política. Actualmente Doctoranda en la Universidad de Vigo en el proyecto de I+D+I (Retos) "DEBATv, Debates Electorales Televisados en España: Modelos, Proceso, Diagnostico y Propuesta" (Ref. CSO2017- 83159-R), financiado por el Ministerio de Economía, Industria y Competitividad (MINECO), la Agencia Estatal de Investigación (AEI) y el Fondo Europeo de Desarrollo Regional (FEDER) de la Unión Europea (UE).

erikaconde@uvigo.es

Orcid ID: https://orcid.org/0000-0002-4898-3689 